\title{
Cryptococcus neoformans en heces de palomas (Columba livia) en Lima Metropolitana
}

\author{
Cryptococcus neoformans in the stools of dove (Columba livia) in metropolitan Lima
}

\begin{abstract}
Ana Huamán 1,2,a, Vilma Béjar ${ }^{1,2, a}$, Gloria Sáez ${ }^{3, a}$, Jose Guevara ${ }^{1,2, b}$, Raul Sevilla ${ }^{2, c}$, Mario Tapia ${ }^{2, b}$, Edith Castillo $^{4, a}$, Esther Valencia ${ }^{1, c}$, Luis Marocho ${ }^{1,2, b}$, Elizabeth Pareja ${ }^{1,2, c}$, Giuliana Romero ${ }^{1,2, c}$, Patricia Abanto ${ }^{2, d}$.
\end{abstract}

\section{RESUMEN}

Objetivos: Investigar la presencia del hongo levaduriforme Cryptococcus neoformans en heces de palomas domésticas (Columba livia). Material y métodos: Estudio descriptivo transversal realizado en la ciudad de LimaPerú. Se recolectaron 300 muestras de heces encontradas en los suelos de parques y hospitales. Además se tomó 30 muestras de las cloacas de algunas palomas capturadas. Resultados: Se aisló el hongo en 47 muestras de las que 7 correspondieron al Cryptococcus neoformans var. neoformans pero no se aisló el hongo en cloaca. Las 7 cepas aisladas tuvieron 100\% de sensibilidad frente a Anfotericin B, Nistatina y Clotrimazol; además presentaron 100\% de resistencia a Fluconazol e Itraconazol y 57\% de resistencia a Ketoconazol. Conclusiones: Cryptococcus neoformans se encuentra presente en heces de palomas en Lima Metropolitana.

PALABRAS CLAVE: Cryptococcus, Columbidae, heces. (Fuente: DeCS BIREME).

\section{SUMMARY}

Objectives: To evaluate the presence of Cryptococcus neoformans in the stools of domestic dove (Columba livia). Methods: Cross-sectional study carried-out in the city of Lima-Peru. 300 stool samples found in the soil of parks and hospital were collected. In addition, 30 sewage samples were also processed. Results: the fungus was isolated in 47 samples, Cryptococcus neoformans var neoformans was isolated in 7 of these samples, none was isolated from sewage samples. The seven strains showed 100\% susceptibility to amphotericin B, nystatin and clotrimazol, but showed 100\% resistance to fluconazole and itraconazole and 57\% resistance to ketoconazole. Conclusions: Cryptococcus neoformansis found in the stools of domestic dove in metropolitan Lima.

KEY WORDS: Cryptococcus, Columbidae, feces. (Source: MeSH NLM).

\section{INTRODUCCIÓN}

Cryptococccus neoformans es un hongo levaduriforme que puede causar micosis oportunista en función del estado inmunológico del paciente (1). Algunas variedades de este hongo, que tiene una distribución mundial, han demostrado predilección por lugares contaminados con excrementos de aves, especialmente de palomas (Columba livia) (1-5).

El hecho de no haber obtenido evidencias suficientes para aceptar la posibilidad que la infección de las personas sea por trasmisión directa entre individuos, favorece la hipótesis que debe adquirirse

\footnotetext{
Instituto de Medicina Tropical Daniel A. Carrión, Facultad de Medicina, Universidad Nacional Mayor de San Marcos. Lima, Perú. Departamento de Microbiología Médica, Facultad de Medicina, Universidad Nacional Mayor de San Marcos. Lima, Perú.

Facultad de Ciencias, Universidad Nacional Federico Villarreal. Lima, Perú.

Dirección Regional de Salud. Callao, Perú.

Biólogo; ${ }^{\mathrm{b}}$ Médico cirujano; ${ }^{\mathrm{c}}$ Tecnólogo médico; ${ }^{\mathrm{d}}$ Técnico de laboratorio
} 
por inhalación de fuentes ambientales (4), entonces, conocer los lugares donde se pueda encontrar el hongo tendrían importante relevancia como posibles fuentes de infección humana $(1,2)$.

Por esta razón, se vio la necesidad de investigar la presencia de Cryptococccus neoformans en lugares de uso común de nuestra ciudad, con presencia de palomas domésticas y sus deyecciones. Especial interés surgió en estudiar su presencia en hospitales, en donde podría encontrarse población especialmente vulnerable, además de algunas áreas verdes de gran extensión.

El objetivo del estudio fue investigar la presencia de Cryptococcus neoformans, en heces de palomas domésticas excretadas y a punto de ser excretadas (en las cloacas), en áreas urbanas y hospitales de Lima Metropolitana, y determinar la sensibilidad antimicótica de los aislamientos.

\section{MATERIAL Y MÉTODOS}

Estudio descriptivo transversal realizado en Lima Metropolitana (Lima-Perú) desde setiembre del 2006 hasta enero del 2007. La ciudad de Lima, capital de Perú, se ubica a una altitud de $101 \mathrm{msnm}$ y tiene una temperatura ambiental que oscila entre $13^{\circ} \mathrm{C}$ en invierno y $28-30^{\circ} \mathrm{C}$ en verano, convirtiéndola en una ciudad costera de clima relativamente templado, con una humedad relativa que varía entre $80-100 \%$ durante todo el año.

\section{Recolección de muestras:}

Se recolectaron muestras de heces de palomas domésticas (Columba livia), en diferentes lugares de Lima Metropolitana: Plazuela de la Iglesia de San Francisco, patios y alrededores del Hospital Nacional Dos de Mayo y patios interiores del Hospital Nacional Arzobispo Loayza (Figura 1).

Se recolectaron 300 muestras de heces, entre secas y frescas, 60 de los hospitales mencionados y 240 de los restantes lugares. Cada muestra fue de por lo menos un gramo, utilizando para su recojo una espátula desinfectada y bolsas plásticas de primer uso rotuladas con los datos del lugar. Asimismo, se constató la densidad de las palomas presentes. Las muestras fueron trasladadas al laboratorio para su procesamiento el mismo día.

Además, en los mismos lugares, se tomaron 30 muestras cloacales con hisopo, de palomas capturadas. Estas muestras fueron transportadas en el medio Amies y sembradas el mismo día en placas con agar Pal (6).

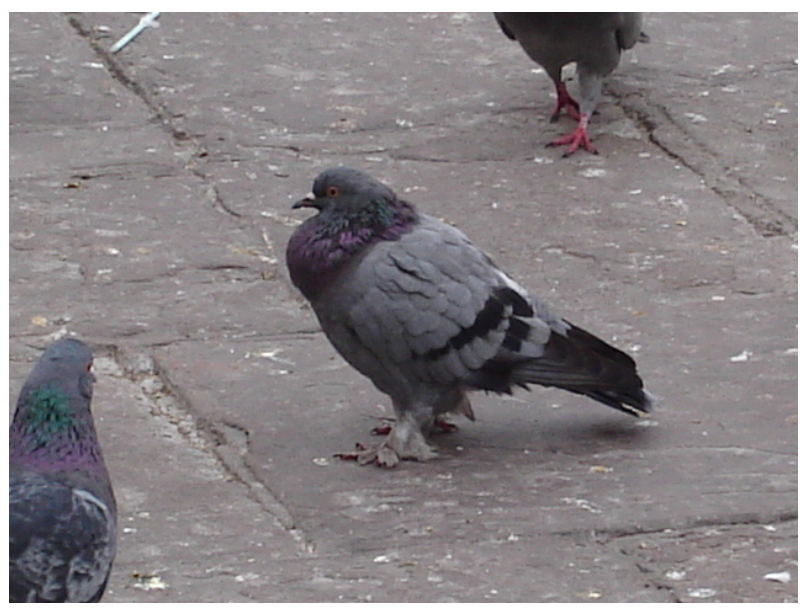

Figura 1. Columba livia en plazuela de Lima Metropolitana

Adicionalmente se tomó algunos datos anexos, como ubicación de la muestra, número de palomas presentes y $\mathrm{pH}$ de las heces (determinadoposteriormente).

\section{Procesamiento de las muestras:}

El procesamiento se realizó modificando ligeramente esquemas ya trabajados en otros estudios (7). Las muestras de heces, secas o frescas, se procesaron individualmente diluyendo un gramo de heces en frascos que contenían $99 \mathrm{ml}$ de solución salina estéril al $0,85 \%$ con cloramfenicol. Se agitó vigorosamente y se dejó reposar unos 15 minutos. Luego se tomó $100 \mathrm{ul}$ de la mezcla, previo homogenizado, y se sembró en placas con agar Pal (6), un medio bioquímico preparado en base a semilla de girasol. Las muestras cloacales también fueron sembradas en agar Pal siguiendo el mismo esquema descrito.

Las placas fueron incubadas a $25^{\circ} \mathrm{C}$ y examinadas diariamente durante 5 días buscando el desarrollo de colonias color café semejantes a las descritas para Cryptococcus. A las colonias sospechosas se les hizo un examen directo con azul de lactofenol y con tinta china para la observación de cápsula, y se resembraron en Agar Sabouraud para la identificación de especie con pruebas diferenciales como hidrólisis de la urea y asimilación de carbohidratos. A las cepas aisladas y catalogadas como Cryptococcusneoformans se les determinó la variedad mediante la prueba de la D-prolina (8).

\section{Evaluación de la sensibilidad antimicótica:}

La sensibilidad a los antifúngicos se determinó por el método de difusión de discos siguiendo el protocolo establecido por la National Committee for 
Clinical Laboratory Standards (NCCLS) documento M44-A (9). A cada cepa aislada se le realizó una suspensión equivalente al tubo $\mathrm{N}^{\circ} 0,5$ de la Escala de McFarland y se sembró (en tapete) en una placa de agar Mueller Hinton con azul de metileno y $2 \%$ de glucosa. Se colocaron discos (HIMEDIA Laboratory Mumbai India) con fluconazol (25 ug), itraconazol (30 ug), anfotericina B (100 unidades), nistatina (100 unidades), ketoconazol (50 ug) y clotrimazol (10ug). Se incubó a $37^{\circ} \mathrm{C}$ por 24 horas antes de proceder a la lectura. El control de calidad se realizó con cepas de Candida albicans ATCC 10231 y Sacharomyces cereviseae ATCC 9763.

\section{RESULTADOS}

En ninguna de las muestras cloacales se aisló Cryptococcus. En 47/300 muestras de heces secas y frescas se aisló Cryptococcussp (Tabla 1). Siete $(14,9 \%)$ aislados correspondieron a Cryptococcus neoformans y todos de la variedad neoformans.

Tabla 1. Especies de Cryptococcus aisladas de heces de palomas.

\begin{tabular}{lc}
\hline Especies de Cryptococcus & n (\%) \\
\hline Cr. laurentii & $23(48,9)$ \\
Cr. uniguttulatus & $3(6,4)$ \\
Cr. gastricus & $4(8,5)$ \\
Cr. albidus & $3(6,4)$ \\
Cr. neoformans & $7(14,9)$ \\
Cr luteolus & $6(12,8)$ \\
Cr. albidus-diffuens & $1(2,1)$ \\
Total & $47(100)$ \\
\hline
\end{tabular}

Se estudiaron muestras de heces de palomas procedentes de zonas urbanas (240) y de centros hospitalarios (60). Cryptococcus neoformans var. Neoformans fueron aislados en seis muestras procedentes de zonas urbanas; 6 de la Plazuela de la Iglesia de San Francisco (3 de muestras secas y 3 de muestras frescas) y una (muestra seca) de los alrededores del Hospital Nacional Dos de Mayo. El $\mathrm{pH}$ en todas las muestras estuvo dentro del rango de 4,5 a 5,0 .

Seis de los siete aislamientos se obtuvieron en muestras procedentes de lugares con más de 15 ejemplares de Columba livia, considerando el valor de 15 como el límite entre alta y baja densidad, concepto tomado de estudios anteriores (5).

Con respecto a la sensibilidad antifúngica, las 7 cepas de Cryptococcus neoformans var. neoformans fueron sensibles a nistatina, anfotericina $\mathrm{B}$ y clotrimazol,y resistentes al itraconazol y fluconazol (Tabla 2).

\section{DISCUSIÓN}

El beneficio de las heces de las palomas en la proliferación de levaduras del género Cryptococcus, referido en estudios anteriores $(5,8)$, también se encontró en nuestro trabajo. El hecho de no aislar el hongo en las muestras de la cloaca, puede significar que las palomas no serían reservorios naturales (10), pudiéndose inferir que las heces favorecerían el crecimiento de levaduras existentes en el ambiente. Existen estudios en los que no se aisló Cryptococcus en heces recién emitidas (11-13).

El saber que menos del $15 \%$ de los aislamientos, fueron Cryptococcus neoformans da cierta tranquilidad pues a la fecha se considera que es la especie patógena para el hombre (4). Además, no llama la atención

Tabla 2. Sensibilidad antifúngica de Cryptococcus neoformans var. Neoformans.

\begin{tabular}{lccccccc}
\hline \multirow{2}{*}{ Antifúngicos } & \multicolumn{7}{c}{ Cepas de Cryptococcus neoformans var. neoformans } \\
\cline { 2 - 7 } & $\mathrm{S} 10-1$ & $\mathrm{~S} 10-2$ & $\mathrm{MS}-2$ & $40-1$ & $\mathrm{Sa} 12-1$ & $\mathrm{M} 27$ & $\mathrm{~S} 12-1$ \\
\hline Fluconazol & $\mathrm{R}$ & $\mathrm{R}$ & $\mathrm{R}$ & $\mathrm{R}$ & $\mathrm{R}$ & $\mathrm{R}$ & $\mathrm{R}$ \\
Nistatina & $\mathrm{S}$ & $\mathrm{S}$ & $\mathrm{S}$ & $\mathrm{S}$ & $\mathrm{S}$ & $\mathrm{S}$ & $\mathrm{S}$ \\
Itraconazol & $\mathrm{R}$ & $\mathrm{R}$ & $\mathrm{R}$ & $\mathrm{R}$ & $\mathrm{R}$ & $\mathrm{R}$ & $\mathrm{R}$ \\
Anfotericina B & $\mathrm{S}$ & $\mathrm{S}$ & $\mathrm{S}$ & $\mathrm{S}$ & $\mathrm{S}$ & $\mathrm{S}$ & $\mathrm{S}$ \\
Clotrimazol & $\mathrm{S}$ & $\mathrm{S}$ & $\mathrm{S}$ & $\mathrm{S}$ & $\mathrm{S}$ & $\mathrm{S}$ & $\mathrm{S}$ \\
Ketoconazol & $\mathrm{R}$ & $\mathrm{R}$ & $\mathrm{S}$ & $\mathrm{S}$ & $\mathrm{S}$ & $\mathrm{R}$ & $\mathrm{R}$ \\
\hline
\end{tabular}


que todos los aislamientos hayan sido de la variedad neoformans pues su afinidad a la creatinina, xantina, urea y ácido úrico, presente en las heces de las palomas (11), seguramente favoreció su crecimiento. Curo (14), encontró hallazgos similares a nuestro estudio en el perímetro urbano de Ica en el 2002.

Las características de las heces, secas o frescas, para algunos investigadores no tienen mayor importancia en el aislamiento del hongo (15); sin embargo, en nuestro estudio hubo cierta predominancia de aislamientos en heces secas (4 de 7 aislamientos), similar a otros estudios (12,13). Asimismo, la mayor cantidad de excretas también parece ser un factor importante porque el mayor número de aislamientos (6 de 7) fue en los lugares con mayor densidad de palomas.

El no haber aislado el hongo en muestras procedentes de hospitales podría deberse al uso de materiales desinfectantes, junto a las actividades de limpieza general que suelen realizarse en los nosocomios, que pueden interferir en la colonización inicial del hongo, situación que no sucede en lugares abiertos y de tránsito ilimitado.

La sensibilidad a la anfotericina B fue similar a otros estudios (16-18), la resistencia al fluconazol fue similar al encontrado en el estudio de García-Martos (16), sin embargo, en este mismo estudio encontraron sensibilidad total al itraconazol y ketokonazol, a diferencia de lo hallado en nuestros aislamientos.

En conclusión, Cryptococcus neoformans variedad neoformans se encuentra presente en heces de palomas halladas en parques recreacionales de Lima metropolitana, todas resistentes a fluconazol e itraconazol. Para limitar la presencia de este patógeno oportunista debería contarse con la limpieza periódica de estas áreas de concurrencia pública.

\section{Declaración de financiamiento y de conflictos de intereses:}

Los autores declaran no tener conflicto de intereses

\section{Correspondencia:}

Ana M. Huamán Reyes.

Correo electrónico: amhr2004@yahoo.es

\section{REFERENCIAS BIBLIOGRÁFICAS}

1. Quintero E, Castañeda E, Ruiz A. Distribución ambiental de Cryptococccus neoformans en el departamento de Cundinamarca-Colombia. Rev Iberoam Micol. 2005; 22:93-98

2. Lobaina P, León A. Meningoencefalitis crónica por Cryptococccus neoformans. MEDISAN. 2004; 8(1): 54-57. (Citado el 23 de julio del 2013) Disponible en: http://bvs.sld.cu/revistas/san/vol8_n1_04/san10104. htm

3. Ayala D, Lopez F, Valencia R. Aislamiento de Cryptococccus neoformans en muestras del ambiente contaminadas con excrementos de palomas en diferentes zonas en El Salvador. Minerva. 2011; 2(1):21-7. (Citado el 23 de julio del 2013) Disponible en: http://www.cic.ues.edu.sv/REVISTA_CIC-UES MINERVA/articulos/Ciencias\%20de $\% 201 \mathrm{a} \% 20$ Salud/criptococcus.pdf

4. Rosario I, Acosta B, Colom F. La paloma y otras aves como reservorio de Cryptococcus spp. Rev Iberoam Micol. 2008; 25:S13-S18

5. Caicedo L, Alvarez M, Llanos C, Molina D. Cryptococccus neoformans en excretas de palomas del perímetro urbano de Cali. Colombia Medica. 1996; 27(3-4):106-109.

6. Al Mosaid A, Sullivan D, Coleman D. Differentiation of Candida dublinensis from Candida albicans on Pal's Agar. J Clin Microbiol. 2003; 41:4787-4789.

7. Pal M. First report of isolation of Crytococcus neoformans var. neoformans from avian excreta in Kathmandu, Nepal. Rev Iberoam Micol. 1997; 14:181-183.

8. Martinez G, Barrial L, Illnait M, et al. Utilidad de la D-prolina en la diferenciación de las variedades de Cryptococccus neoformans. Rev Cubana Med Trop. 2004; 56:77-79

9. Clinical and Laboratory Standards Institute. Method for antifungal disk diffusion susceptibility testing of yeasts. Approved standard M44-A. Wayne, PA: Clinical and Laboratory Standards Institute; 2004.

10. Ordóñez N, Castañeda E. Serotipificación de aislamientos clínicos y del medio ambiente de Cryptococcus neoformans en Colombia. Biomédica.1994; 14:131-139.

11. Ruiz A, Fromtling RA, Bulmer GS. Distribution of Cryptococcus neoformans in a natural site. Infect Immun. 1981; 31:560-563.

12. Kielstein P, Bocklisch H, Hotzel H, Schmalreck A, Otto B. Evidence of Cryptococcus neoformans in domestic and sports pigeons in Thyringia. Mycoses. 2000; 43:23-28.

13. Mattsson R, Haemig PD, Olsen B. Feral pigeons as carriers of Cryptococcus laurentii, Cryptococcus uniguttulatus and Debaryomyces hansenii. Med Mycol. 1999; 37:367-369.

14. Curo M, Salinas M, Casquero J. Cryptococcus neoformans en excretas de palomas, suelo y aire de los palomares del perímetro Urbano de Ica, 2002. Rev Per Med Exp Salud Publica. 2005; 22:262-266. 
15. Castañón LR, Arreguín R, Ruiz G, López R. Frequency of Cryptococcus species and varieties in México and their comparison with some Latin American Countries. Rev Latinoam Microbiol. 2000; 42: 35-40.

16. Garcia-Martos P, Noval J, Garcia-Tapia A, et al. Sensibilidad a antifúngicos de especies de Cryptococcus de interés clínico. Med Clin (Barc). 2002; 119:211-213.
17. Fernandez C, Gonzales M, Illnait T, Martinez G. Determinación de la concentración mínima inhibitoria de anfotericina B en levaduras de interés médico. Rev Cubana Med Trop. 1998; 50:48-53.

18. Catalan M, Montejo J. Antifúngicos sistémicos: Farmacodinamia y farmacocinética. Rev Iberoam Micol. 2006; 23:39-49.

Recibido: $18 / 10 / 2013$

Aceptado: 20/03/2018 